\title{
SARTRE Y LA TRASCENDENCIA DEL EGO: LA PREPARACIÓN DE UNA FILOSOFÍA EXISTENCIAL A LA LUZ DE UNA “ONTOLOGÍA FENOMENOLÓGICA"
}

\author{
Alejandro Escudero Morales \\ Universidad DE Chile \\ ReCEPCIÓN：9.07.2017 ACEPTACIÓN：15.09.2017
}

\section{RESUMEN}

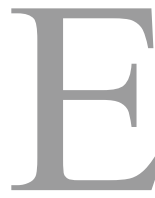

L PRESENTE ARTíCUlo TIENE COMO OBJETIVO SEÑALAR EL PROCEDImiento, que Jean Paul Sartre lleva a cabo en su primera obra filosófica, La trascendencia del ego. A nuestro juicio, esta obra al igual que $\mathrm{El}$ ser y la nada tiene como fundamento metodológico la llamada "ontología fenomenológica". Con el fin de fundamentar esta tesis señalamos que el ego trascendente y el cogito tienen el mismo sentido ontológico que el ser-en-sí y ser-para-sí.

Palabras claves: Sartre, ontología fenomenológica, ego, conciencia, en-sí, para-sí

\section{SARTRE AND THE TRANSCENDENCE OF THE EGO: THE PREPARATION OF AN EXISTENTIAL PHILOSOPHY IN LIGHT OF A "PHENOMENOLOGICAL ONTOLOGY"}

\begin{abstract}
The present article has as aim indicate the procedure that Jean Paul Sartre carries out in his first philosophical work, The transcendence of ego. In our opinion, this work, like Being and Nothing, has as its methodological foundation the so-called "phenomenological ontology". In order to substantiate this thesis we point out that

1 Magister en Filosofía (en curso), Universidad de Chile, 2017. Licenciado en Filosofía, Universidad de Chile, Santiago, Chile. Correo electrónico: a.escudero@live.cl
\end{abstract}


the transcendent ego and the cogito have the same ontological meaning as being-in-itself and being-for-itself.

Keywords: Sartre, phenomenological ontology, ego, conscience, being-in-itself, being-for-itself

Jean Paul Sartre es uno de los filósofos más importantes del siglo XX, no obstante, hoy en día su figura no tiene la relevancia que alguna vez tuvo. Quizás su obra literaria sea bien considerada en nuestro tiempo, pero su filosofía, por lo menos en la academia, dista de tener la importancia que debiera. Con el afán de sumarnos a aquellos que piensan en la valía del pensamiento sartreano es que nos propusimos esta investigación. Si bien es cierto, la obra del francés es extensa, en esta ocasión nos ocuparemos de su primera obra filosófica, La trascendencia del ego. Para esto debemos tener presente que esta es una de las tantas obras de la primera etapa del pensamiento del francés, la cual se caracteriza por ser esencialmente "fenomenológica" y donde se despliegan una serie de cuestiones en torno a la filosofía fundada por Edmund Husserl. A diferencia de La imaginación, de Lo imaginario y de un Bosquejo de la teoría de las emociones, en La trascendencia del ego hay una crítica manifiesta a su maestro. En lugar de apoyar a Husserl y su fenomenología trascendental, el francés rechaza dicha postura y desarrolla una nueva fenomenología. Se ilustra en esta obra de 1934, una nueva forma de abordar la filosofía fenomenológica, distinto de lo que sucede con los otros textos de este periodo, en donde es más cauto respecto del procedimiento promovido por Husserl. De ahí que nosotros la encontremos relevante, puesto que el lugar que ocupa en la filosofía de Sartre es justamente significativo teniendo en cuenta que esa misma actitud respecto de la fenomenología es tratada en su obra capital, El ser y la nada. Frecuentemente se valora esta obra de 1943 como el desarrollo cabal del tipo de procedimiento filosófico por el cual es conocido Sartre, el "existencialismo". La fórmula que propone es la llamada "ontología fenomenológica", donde encontramos como fundamento metodológico, el par en-sí y para-sí. ${ }^{2}$ En esta investigación trataremos principalmente de demostrar que en la obra de 1934 también se hace presente dicho procedimiento, es decir, ya en la primera obra filosófica del francés se encuentra la llamada “ontología fenomenológica”. Para esto nos

2 Existe también el ser-para-otro, al cual dejamos de lado, puesto que nos desviaríamos de nuestro tema. Para más detalle remitirse a la tercera parte del Ser y la nada: El para-otro. 
enfocaremos en esas dos obras y haremos un estudio exhaustivo considerando el ego trascendente y la conciencia a la par del ser-en-sí y ser-para-sí, examen que nos podrá aclarar si efectivamente es el texto de 1934 un estudio donde se hace presente el existencialismo y, asimismo, examinar hasta qué punto es Heidegger el que determina la postura existencial del parisino. En este sentido trataremos de responder a lo largo de la investigación, principalmente estas tres preguntas tentativas: ¿Hay efectivamente en La trascendencia del ego una ontología fenomenológica? ¿Ha sido siempre la postura fenomenológica de Sartre una cuestión de ontología fenomenológica? ¿Es posible suponer que en La trascendencia del ego hay existencialismo?

\section{LA TRASCENDENCIA DEL EGO Y EL EXAMEN DE PRINCIPIOS FENOMENOLÓGICOS Y ONTOLÓGICOS}

Existe una serie de textos de carácter filosófico que anteceden a El ser y la nada: La imaginación (1936), Bosquejo de una teoría de las emociones (1939), Lo imaginario (1940) y La trascendencia del ego, siendo este último el primero de la cronología puesto que fue publicado en $1934^{3}$. Tras acercarnos a la obra filosófica del pensador francés, resulta relevante e imposible de eludir la relación entre La trascendencia del ego y su obra principal, El ser y la nada. En esta última encontramos, al analizar desde la introducción hasta la segunda parte ${ }^{4}$, cuestiones de carácter "metodológico", conceptual, etc., ya presentes en el texto de 1934. Sartre, en esta obra, tiene como objetivo principal estudiar la fenomenología de Husserl, autor alemán que plantea así los fundamentos de esta disciplina: “[...] yo, el fenomenólogo que medita, me propongo a mí mismo la tarea universal de descubrirme a mí mismo como ego trascendental en mi plena concreción y, por tanto, con todos los correlatos intencionales allí incluidos" (Husserl 1986, 53). A diferencia de la tarea que se autoimpone la fenomenología husserliana, Sartre señala que no hay dicho ego trascendental, precisamente porque es la conciencia la que tiene la facultad de ser conciencia de algo: "El Yo trascendental es la muerte de la conciencia" (Sartre 1968, 20). Esta declaración trae consigo dos consecuencias fundamentales para la fenomenología sartreana: por una parte, la conciencia queda

3 Seguimos la cronología hecha por Silvie Le Bon en la Introducción de La trascendencia del ego

4 Introducción en busca del ser. Primera parte: el problema de la nada. Segunda parte: el ser-para-sí 
liberada de una referencia egológica, vale decir, le corresponde un campo trascendental sin ego ${ }^{5}$, y por otra parte el ego no es trascendental sino que trascendente. En este sentido, tal como lo señala Encara González, el francés no elude el rol constituyente que tiene la conciencia: "Para Sartre sí existe una conciencia constituyente; sí está de acuerdo en que existe un mí mismo psíquico y psicofísico. Pero ese mí mismo debe caer bajo el golpe de la epokhé para evitar que se redoble sobre sí mismo convirtiéndose en un Yo trascendental" (González 2007, 111) De esta manera, Sartre señala en este punto que el ego al ser trascendente y no trascendental, es unidad de los estados, de las acciones y las cualidades de la psique. ${ }^{6}$ Como vemos, Sartre en La trascendencia del ego es crítico de las reflexiones de Husserl. Esta postura resulta ser determinante en el pensamiento del francés, no solo en lo que respecta a su primera obra sino también en El ser y la nada. En efecto sobre la base de su crítica al ego trascendental se desprenden su idea de ego y de conciencia, cuestiones que vemos replicadas en su metodología fundamental del existencialismo, a saber, en tanto para-sí y en-sí.

\section{ONTOlogía Y FENOMENOLOGía SARTREANA:}

\section{UNA APROXIMACIÓN CON EL EGO TRASCENDENTE Y EL COGITO DE LA TRAS-} CENDENCIA DEL EGO: EL SER-EN-SÍ Y EL EGO TRASCENDENTE / EL SER-PARA-SÍ Y EL COGITO

Cuando Sartre se refiere al ego lo hace bajo la estricta condición de que sea un objeto trascendente. Llama la atención que pueda concebirlo de esta manera, dado que su explicación considera a filósofos que no comparten la definición que él mismo propone para el ego?. A su juicio, "El Yo es un existente. Hay un tipo de existencia

5 Esta dilucidación particular que Sartre considera acá llama profundamente la atención de Gilles Deleuze quien señala en un artículo de 1995 llamado "La inmanencia: una vida...": "Lo trascendente no es lo trascendental. A falta de conciencia, el campo trascendental se definirá como un puro plano de inmanencia, porque escapa a la trascendencia tanto del sujeto como del objeto". DELEUZE, Gilles. En Ensayos sobre biopolitica. Argentina: Paidós, 2009, pp. 36.

6 No nos detendremos mayormente respecto de este ego trascendente. Por ahora, solo podemos mencionar que es la unidad de los estados, acciones y las cualidades. Para más detalle véase a partir del segundo capítulo de La trascendencia del ego, llamado la "Constitución del ego" donde se desarrolla la concepción del ego sartreano. Desde la página 39 a la 67.

7 Me refiero a filósofos tales como Descartes, Kant, y el mismo Husserl a los cuales Sartre dedica palabras al comienzo de La trascendencia de ego. Véase capítulo, "el Yo y el Yo (moi)" 
concreta, diferente sin duda del de las verdades matemáticas, de las significaciones o de los seres espacio-temporales, pero no menos real. Se da él mismo como trascendente" (Sartre 1968, 30). Bajo la influencia clara de la fenomenología de Husserl, la cita concibe al ego como un objeto trascendente, equivalente a los seres que encontramos cotidianamente en la experiencia, a la naturaleza de las significaciones y también a las verdades matemáticas. Estas "realidades" poseen la particularidad de ser independientes respecto de una experiencia singular. ${ }^{8}$ En este sentido, si somos capaces de entender que el ego al que aludimos debe "aparecer", podemos, ciertamente, demandar algún tipo de acceso una vez que se le confiere el carácter de ser un objeto trascendente. Para Sartre, es en la psicología dónde deberíamos encontrar el lugar para abordar propiamente el fenómeno Ego. De esta manera, la propuesta sartreana guarda relación con una idea de psicología claramente influenciada con el saber fenomenológico. ${ }^{9}$ Para ser más específicos, observemos la siguiente cita.

Lo psíquico es el objeto trascendente de la conciencia reflexiva, es también el objeto de la ciencia llamada psicología. El ego aparece a la reflexión como un objeto trascendente que realiza la síntesis permanente de lo psíquico. El ego está del lado de lo psíquico (Sartre 1968, 49).

En este pasaje, el francés destaca dos cuestiones: por un lado, señala que tenemos acceso al ego gracias a la conciencia reflexiva, vale decir, en estricto rigor lo aprehendemos en la medida que nos encontremos en un acto reflexivo. Por otra parte, como antes señalamos, es un objeto que se considera en la psicología. Cabe destacar, para ser más exhaustivos en la observación, que, si hablamos en términos de reflexión, el ego aparece en la conciencia reflexionada, la cual forma el par conciencia reflexionante-reflexionada que posibilita cualquier acto de reflexión. No se trata de que la conciencia reflexionante contenga el ego, sino que aparece en la conciencia reflexionada; incluso no es parte de ninguna de las dos conciencias, sino que se da a

8 Para más detalle véase Las investigaciones lógicas, 1. Respecto de las significaciones tener en cuenta "la primera investigación: expresión y significación".

9 Sartre en La imaginación, en Lo imaginario y en el Bosquejo de una teoría de las emociones tematiza cuestiones de un orden psicológico a partir de un ejercicio fenomenológico. En La trascendencia del ego también lo pretende, sin embargo, su examen excede dicha pretensión y deviene propiamente en un estudio filosófico, tal como lo intentamos desarrollar en esta investigación. 
través de uno de ellos, en efecto, "[...] no aparece a la reflexión como la conciencia reflexionada; se da a través de la conciencia reflexionada" (Sartre 1968, 29). El ego no es en estricto rigor un fenómeno definible de una manera tradicional, puesto que tenemos conocimientos de él gracias a la reflexión. Pareciera ser que la conciencia reflexionante lo contendría, sin embargo, pensar de esta manera es un error, de hecho, como bien dice la cita, el ego lo intuimos a través de la conciencia reflexionada. En estricto rigor no "se da" como un objeto, sino que debe su ser a otra cosa distinta de sí, en otras palabras, es en la conciencia reflexionada donde se haría posible el "lugar" de su aparición. Independiente de la complejidad de este razonamiento, nos resultaría fácil de comprender si buscamos el modo de ser que Sartre le confiere en El ser y la nada. Conforme a la descripción del ego como un objeto trascendente, el autor lo concibe como un ser-en-sí. En este punto, nos vemos en la obligación de recurrir a su tratado de ontología y fenomenología, ${ }^{10}$ donde se ilustra de manera detallada este modo de ser: "notemos solo la razón de la trascendencia del ego: como polo unificador de las vivencias, el ego es en-sí, no para-sí” (Sartre 2008, 165). Como ya lo señalábamos, el ego es el polo unificador de las vivencias, las cuales tienen una importancia preponderante en la fenomenología husserliana. ${ }^{11}$ Pues bien, en Sartre el ego tiene un carácter de ser-en-sí, es decir, dentro de la empresa filosófica sartreana se debe considerar en este sentido y no como un ser-para-sí. En estricto rigor el para-sí dice relación con la conciencia, en efecto, hablando en términos ontológicos es el ser de la conciencia. Pero no solo esto: Fernando Rodríguez, además de señalar el planteamiento "psíquico" que se extiende desde el principio hasta el final, propone que en La trascendencia del ego estaríamos en presencia de un texto donde efectivamente existe una ontología. En su caso enfatiza que existe ontología, pero ciertamente como una cuestión de "psicología ontológica", precisamente porque el ego estaría en la esfera de lo psíquico. Por consiguiente, se trata de un desarrollo que se prolonga a la psicología y que Rodríguez afirma como "[...] arquitectura ontológica de la psiquis" (Rodríguez 2008, 42). Por nuestra parte enfatizamos que en el texto de 1934 estaríamos propiamente en presencia de un texto de "ontología fenomenológica". De

10 Cuando hablamos de "tratado de fenomenología y ontología" queremos decir El ser y la nada

11 El ego como polo de las vivencias es examinado por Husserl fundamentalmente en las Meditaciones Cartesianas. Cabe mencionar que no nos detendremos mayormente en Edmund Husserl puesto que nos alejamos de nuestro propósito de investigación. 
todos modos, es nuestra obligación acudir a Sartre para dilucidar qué quiere decir cuando se refiere al ser-en-sí: "El ser es. El ser es en sí. El ser es lo que es. He aquí las tres características que el examen provisional del fenómeno de ser nos permite asignar al ser de los fenómenos" (Sartre 2008, 38). Por ser-en-sí entendemos todo aquello que existe, excluyendo de esta denominación a la conciencia del hombre ya que el ser de esta es el para-sí. Cuando nos referimos a todo aquello que existe hablamos de los fenómenos que tienen un tipo de ser que el filósofo denomina por ser-en-sí. En la cita queda claro que todo ser de los fenómenos se debe a tres características, lo que conduce a sostener que el ser de los fenómenos no puede ser de otra manera que no sea en-sí. Pareciera ser una cuestión que no acepta dudas sobre su naturaleza, puesto que es lo que es y no puede ser otra cosa, es más, el propio filósofo lo propone como, “[...] opaco a sí mismo precisamente porque está lleno de sí mismo” (Sartre 2008, 36), es decir, por su modo de ser, es inobjetable gracias a la identidad que lo convierte en ser lo que es sin ninguna remisión a otra cosa, señalándolo además de opaco como macizo ya que no le es posible guardar secretos respecto de su ser. ${ }^{12}$

Que el ego sea un ser-en-sí quiere decir que su determinación, por lo menos en Sartre, se debe a que tiene un tipo de ser, igual a todos aquellos que no concierne a lo absoluto, es decir, a la conciencia. Efectivamente, es considerado bajo un mismo criterio que los seres espacio-temporales. Habría, por lo tanto, una alusión a este ser-ensí ya concebido en La trascendencia del ego, precisamente cuando se cataloga al ego como un objeto trascendente, de hecho, es posible constatarlo en la primera cita del presente capítulo, donde en alguna medida se lo concibe a la par de las significaciones, de las verdades eternas, etc. ${ }^{13} \mathrm{Al}$ respecto, cabe destacar que el ego trascendente en tanto objeto trascendente tiene el mismo sentido que el ser-en-sí, en efecto, es el primer objeto analizado por Sartre de ser ontológicamente un en-sí. En este punto es fundamental señalar la importancia de La trascendencia del ego, de hecho, para todo aquel que se quiera introducir en el pensamiento de Jean Paul Sartre es pertinente acercarse, por lo menos en lo que respecta a la empresa filosófica que se origina en 1934, hasta El ser y la nada.

12 Para más detalle véase el capítulo primero. VI. "Ser en sí"

13 Específicamente en la página 4. 
Ahora bien, si podemos examinar al ego trascendente como un ser-en-sí ¿es posible hacer este paralelo con la conciencia, es decir, con el cogito y el ser-para-sí? Trataremos de resolverlo más adelante, pero antes debemos desviarnos un tanto debido a que el ego, desde la empresa sartreana, no solo se establece como un en-sí, sino que también como un "fenómeno" temporal, es decir, le concierne naturalmente una temporalidad. Hablábamos anteriormente de que el ego aparece siempre y cuando se trate de un acto reflexivo, reflexión que tiene dos maneras de ser, pura o impura (constituyente), esta última definida en tanto que "[...] constituye la sucesión de hechos psíquicos o psique" (Sartre 2008, 233). En este sentido, bajo esta nueva observación que Sartre propone en el marco de su tratado de ontología fenomenológica, nos encontramos con una nueva manera de acceder al ego del cual afirma su ser trascendente. Esto implica el contraste con la reflexión pura respecto de la reflexión impura, la que en tanto temporalidad es lo que somos pero no en términos psíquicos sino que propiamente ontológicos, esto es, a saber en términos de temporalidad debido a que en el pensamiento sartreano existe una descripción de lo que somos efectivamente como temporalización. ${ }^{14}$ De todas maneras, no nos debemos alejar de nuestro propósito, por lo cual tenemos que quedarnos con la reflexión que nos convoca cuando debemos referirnos al ego. A diferencia de la reflexión pura que en estricto rigor es ser para-sí en tanto par reflexivo-reflexo que no deviene nunca un en-sí, ${ }^{15}$ en la reflexión impura se da el fenómeno "contrario", por lo menos en algún sentido, puesto que lo reflexo deja de ser parte de la remisión infinita entre lo reflexivo-reflexo del para-sí y se descubre como un ser-en-sí, expresado en lo que sigue: "[...] la reflexión impura, que es el movimiento reflexivo primero y espontaneo (pero no originario) es para-ser lo reflexo como en-sí” (Sartre 2008, 234). A pesar de ser la reflexión impura una cuestión previa ya que es lo primero que se da como fenómeno en términos propiamente reflexivos precisamente cuando hablamos del ser pensante que somos, en este punto no estaríamos en presencia de un fenómeno originario ya que esta definición corresponde propiamente a la reflexión pura. De todas formas, si nos referimos a la reflexión impura consideramos la cuestión a propósito del ego en términos del tipo de temporalidad que le constituye. Ahora

14 Para más detalle véase El ser y la nada capitulo segundo. III. "Temporalidad original y temporalidad psíquica".

15 Véase El ser y la nada. P. 233-234. 
bien, solo nos es posible examinar lo reflexo de la mano de Sartre como un en-sí, puesto que lo reflexivo lo toma como tal, constituyéndose con esto tres formas que definen la reflexión impura, ya sea como "[...] lo reflexivo, lo reflexo y un en-sí que lo reflexivo tiene-de-ser en tanto que este en-sí sería lo reflexo y que no es sino el para del fenómeno reflexivo" (Sartre 2008, 234). Al ser el par reflexivo-reflexo un fenómeno que se da siempre en la medida que se conforme la pareja, podríamos hablar independiente de su ser, de un fenómeno separado, precisamente si examinamos lo reflexo como un ser-en-sí, lo que enfatizaría aún más la definición de la reflexión impura respecto de la reflexión pura a propósito de que no es un ser en sentido lato, sino que un ser-sido, ${ }^{16}$ en otras palabras, en un ser que ya fue y del que tenemos conocimiento precisamente porque es en el modo del ser pasado, por lo cual se trataría de un objeto para lo reflexivo, es decir, se toma conciencia de lo reflexo pero como algo, como un en-sí y no como un para-sí. Debemos dejar claro que lo reflexo, en estricto rigor, no se convierte en ser-en-sí, sino que se da detrás de lo reflexo, principalmente porque el par reflexivo-reflexo no es posible detenerlo. Esto quiere decir que el ser trascendente es "[...] una sombra proyectada de lo reflexo [...]" (Sartre 2008, 234) en la medida en que la reflexión "trate" lo reflexo como objeto y no como se da el fenómeno en una reflexión pura. Con este argumento el propio Sartre determina: "El acto por el cual la reflexión se determina a tomar lo reflexo como objeto es, en sí mismo: $1^{\circ}$ posición de lo reflexivo como no siendo lo reflexo; $2^{\circ}$ adopción de un punto de vista con respecto a lo reflexo" (Sartre 2008, 235). Sartre describiría por una parte la reflexión impura bajo condición que lo reflexivo no sea lo reflexo ya que el par siempre se debe dar junto siendo lo reflexivo lo reflexo y viceversa, determinación que no ocurre en la reflexión que definimos y constituimos en condición del dominio psíquico. Por otra parte, estaríamos, en este sentido, en presencia de la perspectiva o punto de vista que toma lo reflexo como una cuestión propiamente "objetiva" lo que permitiría y condicionaría la "separación” de la pareja reflexivo-reflexo. Por de pronto, la sombra que aparece en lo reflexo es el hecho psíquico que el psicólogo, a juicio de Sartre, debe estudiar puesto que estaríamos en presencia del ya nombrado dominio "egológico", pero ahora en

16 Para más detalle véase El ser y la nada. Capitulo II "la temporalidad", donde se definen los modos de ser en tanto pasado, presente y futuro. Cuando hablamos de un ser-sido queremos decir que es posible definirlo puesto que es opaco para el sujeto, no así el ser presente y el ser futuro, ya que son cuestiones que todavía no son y por consiguiente indefinibles. 
un sentido propiamente sartreano no remitiéndose en ninguna medida al razonamiento de Edmund Husserl. ${ }^{17}$ Asimismo, el filósofo francés señala que "la reflexión es impura cuando se da como intuición del para-sí en en-sí” (Sartre 2008, 225) tal y como lo veníamos esbozando en tanto tiene el carácter de ser un "objeto", como algo posible de ser definible, dicho de otra forma, en el mismo sentido de una definición o descripción de una cosa con la cual nos topamos en la cotidianidad, pero en vez de ser una cuestión real o que existe, nos referimos ahora a aquello que tiene el carácter de virtualidad, ${ }^{18}$ como seres que son en-sí pero correspondientes a, "La vida psíquica o psique [...]" (Sartre 2008, 236).

Destacando la labor emprendida por Silvie Le Bon, cuya introducción y notas expuestas detalladamente en cada parte que merecía una revisión en La trascendencia del ego, es que nos vemos en la obligación de acudir a ella, principalmente por su capacidad de síntesis que hace posible una mejor lectura de tan difícil texto para aquellos que no tienen conocimiento de Sartre. De hecho, es necesario tenerla en cuenta por lo menos para hacer de la lectura un ejercicio más ameno y no tan oscuro, considerando el contenido "rígido" del texto publicado en 1934. De este modo, reflexionemos junto a ella, lo siguiente.

Para resumir, un análisis fenomenológico de la conciencia discernirá [Sartre] tres grados de la conciencia: $1^{\circ}$ ) un primer grado al nivel de la conciencia irreflexiva, no posicional de sí, porque es conciencia de sí en tanto que conciencia de un objeto trascendente (Sartre 1968, 90).

Respecto a este grado de conciencia, que no cae en la denominación del cogito, el filósofo oriundo de Paris, afirma "[...] una prioridad ontológica sobre lo reflexivo" (Sartre 1968, 35). Al respecto, Sartre señala que no habría necesidad de pasar al cogito, si consideramos que lo necesario es la conciencia irreflexiva, la que es

17 Husserl fundamenta su egología en tanto hay un ego trascendental fundamento del conocimiento y por ende de todo cuanto exista. Les es inherente a esta posición un ego puro, residuo fenomenológico y asimismo lógico, que en su estado de pureza es la esencia del ego fenomenológico husserliano. Para más detalle remitirse a la posición de Edmund Husserl principalmente en la Meditaciones Cartesianas.

18 Virtualidad propuesta en el mismo sentido que los estados, acciones y cualidades constituyentes del ego trascendente. Véase la "Constitución del ego" en La trascendencia del ego. 
conciencia de sí en tanto conciencia de un objeto trascendente. Una vez que se efectúa un acto reflexivo pasamos al cogito, es decir, al segundo y al tercer grado de la conciencia, cuestión que la hija adoptiva de Simone de Beauvoir, analiza en la siguiente cita:

Con el cogito: $2^{\circ}$ ) un segundo grado: la conciencia reflexionante es no-posicional de sí misma, pero posicional de la conciencia reflexiva. $3^{\circ}$ ) un tercer grado, que es un acto tético en un segundo grado, por el cual la conciencia reflexionante deviene posicional de sí (Sartre 1968, 90).

Para Sartre cuando hablamos de cogito existe un cambio en la conciencia misma, en sus modos de ser, ya sea de no posicional a posicional. De todas maneras, la estructura, más allá de que es difícil de asumir, no podría ser más clara que lo expuesto por Le Bon. Bastaría observar que la conciencia reflexionante una vez que se vuelve posicional de sí, se sostiene como un acto reflexivo, o mejor aún, en tanto cogito mismo. Cabe destacar que nos referimos a la estructura interna de la conciencia, que cambia de un grado a otro en sí misma.

En contraste con el cogito concebido como una cuestión que remite al ego bajo la tradición filosófica en la cual se halla Husserl, Sartre, lejos de razonar respecto de sus antecesores, defiende su posición dejando de lado esta suerte de implicancias entre el ego y el cogito. Es indudable que su empresa provoca tanto en la filosofía y psicología un remesón algo atrevido si "separa" al ego del cogito. En este sentido, el cogito "subsistiría" por sí solo sin necesidad de remitirse a un ego, cuestión que tratábamos de examinar en el razonamiento anterior donde veíamos que no habría necesidad de la reflexión sino solo del dominio irreflexivo constituyente del mundo y de todo cuanto existe. No cabe duda de que Sartre es un fenomenólogo si se refiere al cogito o conciencia, pero claramente bajo una nueva elaboración en donde el papel del ego pareciera ser secundario o posterior al papel primordial y absoluto de la conciencia.

Ahora bien, Sartre se refiere al cogito o conciencia desde el principio del Ser y la nada, a propósito de una observación exhaustiva que emprende, lo cual permite una caracterización en un sentido propiamente ontológico, es decir, en tanto ser-para-sí, 
u ontología fenomenológica. Sartre en este respecto, tiene bastante presente lo que en su texto de 1934 sostuvo, por ejemplo, respecto de la conciencia posicional. De hecho, así lo muestra la siguiente cita.

\begin{abstract}
Así, habremos definido la reflexión o sea la conciencia posicional de la conciencia o, mejor aún, el conocimiento de la conciencia. Sería una conciencia completa y dirigida hacia algo que no es ella, es decir, a la conciencia refleja. Se trascendería, pues; y, como la conciencia posicional del mundo, se agotaría en el apuntar a su objeto. Solo que este objeto sería a su vez una conciencia (Sartre 2008, 19).
\end{abstract}

Como vemos, la conciencia posicional se dirige a algo que ella no es, es decir, a lo trascendente, al mundo. La conciencia a la cual se dirige la conciencia posicional o reflexiva, es la conciencia refleja, que tiene la tarea de reflejar algo, vale decir, el mundo. En estricto rigor, para la reflexión el objeto es la conciencia refleja. Sería la fenomenología sartreana un rescate de todas aquellas filosofías "alimentarias", las que hacen el intento de introducir el mundo en el sujeto, en tanto se habla en términos de "contenidos de conciencia". 19 De todas maneras, con anterioridad a cualquier eventualidad de reflexión, Sartre es claro en hablar sobre lo no-reflexivo, que ya se hace presente en La trascendencia del ego, pero que aquí se lo describe como un cogito prerreflexivo, el que es condición de cualquier reflexión, ilustrado en las propias palabras del alguna vez director de la revista "Tiempos modernos", cito.

[...] la reflexión no tiene primacía de ninguna especie sobre la conciencia refleja: ésta no es revelada a si misma por aquella. Al contrario, la conciencia no-reflexiva hace posible la reflexión: hay un cogito prerreflexivo que es la condición del cogito cartesiano (Sartre 2008, 21).

Si bien es cierto, nos habíamos alejado en alguna medida de aquel primer grado de la conciencia cuya necesidad es constitutiva de la existencia, es decir, de la conciencia irreflexiva, nos vemos en la obligación de aclarar su modo de ser. Sartre expresamen-

19 Para más detalle, véase el primer párrafo de un breve artículo de Sartre llamado "Una idea fundamental de la fenomenología de Husserl: la intencionalidad" en El hombre y las cosas (Situaciones 1) 
te la condiciona como conciencia no posicional de sí, en la cual no habría en estricto rigor, un acto reflexivo, lo cual no determina que no sea conciencia de sí misma. De hecho, la conciencia no posicional de sí, es efectivamente conciencia de sí misma, en tanto objeto trascendente. Veamos en palabras del propio Sartre, lo que quiere decir, de esta conciencia de sí, teniendo en cuenta lo visto de La trascendencia del ego.

Esta conciencia (de) sí no debe ser considerada como una nueva conciencia, sino como el único modo de existencia posible para una conciencia de algo. Así como un objeto extenso está obligado a existir según sus tres dimensiones, así también una intención, un placer, un dolor no podrían existir sino como conciencia inmediata (de) sí mismos (Sartre 2008, 21-22).

De cara al hecho de que tenemos conciencia de un objeto trascendente, es solo posible si la conciencia es conciencia de algo, distinto de sí misma, ella es trascendencia hacia el mundo, en tanto es intencional. Haciendo alusión a su ontología y fenomenología, Sartre reproduce la conciencia en términos de ser, es decir, tiene en su ser otro ser, ya que es conciencia de un objeto trascendente, distinto de sí mismo. Necesariamente debemos ilustrar su pensamiento debido a su complejidad, pero si tenemos en cuenta lo anterior se nos hará mucho más fácil de digerir; “[...] la conciencia es un ser para el cual en su ser es cuestión de su ser en tanto que este ser implica un ser otro que él mismo" (Sartre 2008, 32). Se trataría, por lo tanto, del ser-para-sí, en tanto es un ser que no es el mismo, que en estricto rigor no coincide consigo mismo, vale decir, es un ser que no es idéntico, puesto que esta denominación convendría al ser-en-sí al que ya le dedicamos unas palabras. No podemos dejar pasar, en este estudio, el "sí" del ser-para-sí, que es condición del modo de ser de la conciencia. En este sentido, el "sí" aludido remite al sujeto, a la relación que tendría consigo mismo, a saber, a una distancia de carácter ideal, distancia que no podemos abordar si no es diseñada como una cuestión idealmente sin necesidad de buscar en la experiencia su motivación, de hecho estamos hablando en términos de inmanencia, en otras palabras, de la estructura de la conciencia que el propio Edmund Husserl no se cansa de observar y detallar rigurosamente en Ideas $1 .{ }^{20}$ En este sentido, habría una suerte

20 Se refiere a esta "estructura" de la conciencia a lo largo de Ideas 1 pero específicamente a partir de la sección tercera: "Sobre la metodología y la problemática de la fenomenología pura". Desde la página 221. 
de presencia que la conciencia, en términos de ser-para-sí tiene consigo, de ser presencia a sí, que se dilucida en términos ontológicos de la siguiente manera: "La ley de ser del para-sí, como fundamento ontológico de la conciencia, consiste en ser él mismo en la forma de presencia a sí" (Sartre 2008, 133). Por lo tanto, si concebimos al ser-para-sí en la forma de presencia a sí, lo debemos considerar a propósito de una nada que es constituyente del modo de ser de la conciencia, en tanto supone una distancia en ella misma, determinada por algo que en estricto rigor no es y que se da cuenta en la inmanencia de la conciencia, como una distancia ideal o "nada", que no puede por definición hacer coincidir.

En este sentido La trascendencia del ego sale al rescate respecto de la complejidad del argumento sartreano, puesto que tratan la misma temática, pero naturalmente con otro tipo de metodología y conceptualidad. Debemos dejar claro que, si bien es posible seguir con este examen de constatar si existen relaciones de algún tipo entre La trascendencia del ego y El ser y la nada, hay una "huella" en aquel texto que nos permite sostener la ontología fenomenológica de una mejor forma. Es necesario tener en cuenta las revisiones que hemos hecho ya que es la conciencia en tanto existencia lo que nos permitiría acceder de mejor manera a la finalidad de la presente investigación que trataremos de fundamentar en el siguiente capítulo.

\section{La posibilidad de Pensar La trascendencia del ego Como UNA OBRA DE "ONTOLOGÍA FENOMENOLÓGICA"}

Si pretendemos considerar La trascendencia del ego como una obra de ontología fenomenológica, debemos desplegar los siguientes tres ejes: en primer lugar, ver la posibilidad de determinarla como una ontología fenomenológica. En segundo lugar, examinar la posibilidad de que en el texto de 1934 haya filosofía existencial o, mejor aún, existencialismo. Y en tercer lugar ilustrar el rol que tiene Heidegger respecto de la fenomenología sartreana en su primera obra filosófica.

En primer lugar: ¿es la ontología fenomenológica un procedimiento que se encuentra en la primera obra filosófica de Jean Paul Sartre? En efecto, podemos afirmar esta 
posibilidad si tenemos en cuenta que el en-sí y el para-sí serían desde un comienzo el ego trascendente y la conciencia, tal como quedó demostrado a lo largo de esta investigación. Asimismo, el propio filósofo francés destaca el hecho de que en $L a$ trascendencia del ego se sigue una línea metodológica con El ser y la nada: "Hemos tratado de mostrar en un artículo de las "Recherches philosophiques", ${ }^{21}$ que el ego no pertenecía al dominio del para-sí" (Sartre 2008, 165). Y para subrayar aún más esta línea, el francés afirma: "el ego aparece a la conciencia como un en-sí trascendente, como un existente del mundo, no como de la conciencia" (Sartre 2008, 166). Ahora bien, si tenemos esto en cuenta, es posible señalar, como ya lo habíamos hecho durante este estudio, que el ego trascendente es el primer ser-en-sí analizado por el filósofo francés, en efecto, esto resalta aún más el vínculo entre las obras. Pero, ¿qué consecuencia tiene que el par ser-en-sí y para-sí se encuentre ya en La trascendencia del ego? Es posible afirmar con esto que Sartre desde un comienzo intuía sobre lo que sería su pensamiento filosófico y, por lo tanto, su concepción ontológica del Ser. Obviamente hay diferencias entre cada una de las obras, las cuales nosotros precisamos de la manera siguiente: la diferencia de uno y otro texto se debe al tratamiento que tiene el francés respecto de la fenomenología, a saber, en La trascendencia del ego hay una ontología fenomenológica implícita y en El ser y la nada una ontología fenomenológica explicita.

Siguiendo la línea de la investigación ¿podríamos, en estricto rigor, sostener que en La trascendencia del ego se hace presente la filosofía existencial o existencialismo, teniendo en cuenta que tendría metodológicamente el mismo procedimiento? Efectivamente, Sartre alude ya en la obra de 1934 a esta posibilidad, sobre todo cuando formula su posición respecto a la conciencia, en detrimento de la formulación que propone el padre de la fenomenología. ${ }^{22}$ En Sartre desde un comienzo se vislumbra una conciencia en términos de existencia, en efecto, señala: "[...] la conciencia es un ser cuya esencia implica la existencia" (Sartre 1968, 59). Su manera de abordar su tesis existencial proviene de su revisión y estudio sobre la conciencia trascendental

21 El artículo aparecido en esa revista es, efectivamente, La trascendencia del ego.

22 Si bien es cierto los dos filósofos afirman que la conciencia es conciencia de algo, vale decir, destacan la "intencionalidad de la conciencia", el francés sostiene su posición en tanto la conciencia "existe", en cambio en el alemán la conciencia es fundamento del conocimiento científico. 
husserliana y todo lo que esto conlleva. La fenomenología sartreana u ontología fenomenológica reinterpreta una serie de cuestiones que forman parte de la fenomenología de Husserl. Bajo este punto, podemos señalar, por ahora, su concepción sobre la epojé, que como recurso es una posibilidad que siempre se puede ejecutar, independiente de su determinación "metodológica": “[...] la epojé no es más un milagro, no es más un método intelectual ni un procedimiento del sabio: es una angustia que se impone a nosotros y que nosotros no podemos evitar, es a la vez un acontecimiento puro de origen trascendental y un accidente siempre posible de nuestra vida cotidiana" (Sartre 1968, 78). Consideramos pertinente destacar sus observaciones respecto de la conciencia irreflexiva e impersonal, en cuanto ya no es perteneciente a un ego puro, desde donde se origina. ${ }^{23}$ Respecto de la originalidad de su propuesta fenomenológica Le bon señala:

Sartre siempre insistirá sobre esta autonomía de la conciencia irreflexiva que encuentra su fundamento en la intencionalidad esencial de las conciencias. Esta concepción de la prioridad ontológica de lo irreflexivo sobre lo reflexivo quedará como algo central en sus obras ulteriores, en particular en La imaginación (la imagen es una evidencia ante-predicativa), en la Teoría de las emociones, Lo imaginario y El ser y la nada, porque ella constituye el único medio radical de eliminar todo idealismo (Sartre 1968, 93).

Esto pretende subrayar la conciencia irreflexiva con una notoria relevancia ontológica por sobre lo reflexivo, fundamento del propósito concreto de Sartre, a saber, su anti-idealismo y por lo tanto su objetivo existencial. Se demuestra con esta "tesis" que independiente de su relación con Husserl y Heidegger, el francés ya consideraba su metodología, quizás no en sentido estricto, pero si implícitamente. Es por esto que es común que se tenga en conocimiento que El ser y la nada es una obra de maduración del autor, precisamente porque su pensamiento maduró, se perfeccionó. En efecto, se halla su procedencia metodológica en La trascendencia del ego gracias al estudio que realiza sobre el ego trascendente y conciencia. No podríamos decir lo mismo de

23 Para más detalle véase página 79 y su concepción de epojé que nosotros destacamos como existencial. Respecto de la conciencia trascendental e impersonal liberada y purificada, remitirse desde la página 69 en a delante de La trascendencia del ego. 
sus obras propiamente "fenomenológicas", a saber, La imaginación, Lo imaginario y El bosquejo de una teoría de las emociones, puesto que en ellas hay un ejercicio fenomenológico, más no de ontología fenomenológica.

Con esto, no solo tenemos que La trascendencia del ego es un texto esencialmente de ontología fenomenológica, sino que también podemos remitirnos a la relación intelectual que el propio francés mantiene con Martin Heidegger, filósofo al que reiteradamente se le señala como el promotor del existencialismo alemán. Efectivamente, Sartre se remite a él en El ser y la nada. En este sentido Muñoz, un comentador de la obra de Sartre considera:

Ya desde el subtítulo quiere ser un «Ensayo de ontología fenomenológica». La idea, ciertamente, no es novedosa y tiene como antecedente claro la Ontología fundamental, de Heidegger, hasta el punto que, puede afirmarse, en la introducción a El Ser y la Nada; Sartre nos plantea el mismo objetivo que el filósofo alemán: hallar el sentido del ser (Muñoz 1987, 72).

En este sentido, son evidentes las infinitas referencias que se encuentran respecto del autor del Ser y tiempo, de hecho, su concepción de la conciencia o ser-para-sí tiene directa relación con lo que Heidegger señala respecto del Dasein:

Ciertamente, podríamos aplicar a la conciencia la definición que Heidegger reserva para el Dasein, y decir que es un ser para el cual su ser está en cuestión en su ser, pero sería menester completarla y formularla más o menos así: la conciencia es un ser para el cual en su ser esté en cuestión su ser en tanto que este ser implica un ser diferente de él mismo (Sartre 2008, 32).

A juicio de Sartre, el Dasein heideggeriano supone la misma determinación acerca de su concepción de conciencia. Sin embargo, el francés enfatiza su alejamiento desde que señala que la posición de Heidegger es incompleta, precisamente porque, como vimos antes, el ser de la conciencia es un ser que implica un ser distinto de sí. Pero a pesar de la lejanía que subraya el propio Sartre con relación al alemán, no puede eludir cuestiones tan importantes como las reflexiones que despliegan en el 
Ser y tiempo. Es por esto que para sus planteamientos existenciales requiere de una reflexión tan fundamental como la que implica el ser-en-el-mundo: "Lo concreto es el hombre en el mundo, con esa unión especifica del hombre con el mundo, que Heidegger, por ejemplo, llama «ser-en-el-mundo»" (Sartre 2008, 42). Sartre retoma de Heidegger la relación primordial que tiene el Dasein acerca del mundo, esa relación cuyo fundamento para el francés queda señalado por el ser-para-sí.

Ahora bien, respecto al nexo que Sartre mantiene con Heidegger debemos necesariamente preguntarnos lo siguiente: ¿La tesis existencial de La trascendencia del ego se debe fundamentalmente a la influencia de Heidegger? Si tenemos en cuenta que en El ser y la nada se despliegan los fundamentos de la filosofía existencial, el vínculo que mantiene con sus primeras obras es directo, en efecto tal como Muñoz señala, "La obra El Ser y la Nada, que como es sabido es la coronación de las investigaciones filosóficas emprendidas por Sartre desde 1933 [...] (Muñoz 1987, 71), vale decir, en las entregas que preceden a su obra de 1943, el francés ya señalaba cuestiones que posteriormente iba a desarrollar a cabalidad. Con esto es posible considerar que Heidegger de alguna manera se hacía presente desde un comienzo en la filosofía sartreana, efectivamente, si tenemos en cuenta que la ontología fenomenológica del Ser y la nada tiene presente la ontología fundamental del Ser y tiempo.

Ahora bien, otra visión al respecto que no se condice con la postura de Muñoz es la que propone la destacada filósofa chilena Carla Cordua respecto de la relación que mantiene el francés con Heidegger en su texto, Gerencia del tiempo:" "Sartre no pudo terminar de leer Sein und Zeit durante su estadía en Berlín, en 1934 [...] Llega, en efecto, a leer unas cincuenta páginas en esa ocasión, dice, pero pronto abandona provisoriamente su plan de estudiar a Heidegger" (Cordua 1994, 113). Asimismo, Cordua señala: "[...] cuando finalmente lee el libro en 1939 descubre que ha superado esta dificultad sin haber progresado entretanto en su conocimiento del alemán" (Cordua 1994, 114). La filósofa chilena destaca el hecho de que Sartre en la época en la que escribe no solo La trascendencia del ego, también las demás obras fenomeno-

24 En esta investigación solo ocuparemos el capítulo llamado: “Sartre lee a Heidegger". Para quien se introduzca en el pensamiento de Jean Paul Sartre es recomendable este texto, que contiene una variedad de ensayos sobre el pensador francés. 
lógicas, no se propuso una lectura exhaustiva del alemán, sino que solo hacia 1939 tendría dicha motivación, ya que para esa fecha Sartre ya era un lector de la lengua alemana. En la época en que escribe La trascendencia del ego, el parisino era un fenomenólogo de la tradición husserliana, así lo muestra Cordua:

El llamado periodo husserliano de Sartre dura poco más de unos cuatro años. Durante él Sartre escribe, salvo por los capítulos finales, $L^{\prime}$ imaginaire y " $\mathrm{La}$ trascendence de'l ego" e inicia, en 1937, la redacción de un gran libro, $L a$ psyche que queda inconcluso, pero una de cuyas partes aparece bajo el título de Esquisse d une théorie des émotions (Cordua 1994, 115).

De hecho, si sabemos que El ser y la nada es una obra de 1943 y Sartre comienza en 1939 su lectura del Ser y tiempo, podemos intuir que la obra capital del francés tiene una influencia clara de Heidegger. En efecto, Cordua clasifica la obra Sartreana en tres periodos: "La obra filosófica anterior sería 'fenomenológica'; El ser y la nada, 'heideggeriano' y la obra posterior, 'marxista”' (Cordua 1994, 119). Es posible señalar con lo visto que para aquella época fenomenológica el francés aún no se dedicaba expresamente a estudiar a Heidegger, al contrario, su atención estaba destinada al pensamiento fenomenológico. El énfasis que estamos tratando de considerar tiene relación con el hecho de que en la primera etapa del pensamiento sartreano no hay una influencia explicita del alemán. Quizás podemos considerar que en un primer momento recurrió a Kierkegaard para su tesis existencial, pero su originalidad en cuanto existencialismo fenomenológico o fenomenología existencial es producto de su propia filosofía y no de una influencia directa de Heidegger. Efectivamente el francés afirma: "No podía llegar a Heidegger más que después de haber agotado a Husserl" (Cordua 1994, 114). Con estas reflexiones tenemos la oportunidad de abordar a Sartre desde otra mirada que no tiene estricta relación con Heidegger. Asimismo, podemos considerar que el existencialismo que se despliega en su tratado capital ya se hacía presente en su primera obra filosófica. Bajo este respecto tenemos el privilegio de observar que las reflexiones en torno al existencialismo se encontraban en un origen en él, de alguna manera ya intuía lo que posteriormente iba a hacer su proyecto filosófico, pero aun no tenía las herramientas intelectuales para fundamentarla. En este sentido el pensamiento de Heidegger significa para Sartre 
un pensamiento que influyo en la segunda etapa de su vida intelectual, periodo de un pensamiento ya maduro y no en maduración. Por consiguiente, es posible señalar una nueva forma de pensar la influencia del alemán en Jean Paul Sartre y junto con esto enfatizar que la filosofía sartreana tiene en La trascendencia del ego su origen argumentativo y metodológico.

\section{Conclusión}

El presente artículo pretendía, fundamentalmente, demostrar que Sartre en La trascendencia del ego emprende su obra con la metodología que se encuentra desplegada en El ser y la nada. Esto a raíz de la crítica hacia Husserl y la concepción de ego trascendental, asunto que trae consigo la distinción entre el ego trascendente y la conciencia. Se desprende de esta distinción que Sartre desde la génesis de su pensamiento se apoyaba en principios ontológicos, principalmente porque el ego trascendente y la conciencia tienen como fundamento ontológico el en-sí y para-sí. En este punto es menester recalcar que el ego trascendente es el primer ser-en-sí desarrollado por Sartre, cuestión de una valía incalculable si pensamos que el en-sí es un elemento primordial para la filosofía existencial. La temática se profundizó una vez que efectivamente encontramos presupuestos existenciales. En efecto, Sartre se encuentra en un periodo fenomenológico, llamado también periodo Berlinés, ${ }^{25}$ donde su interlocutor principal es Husserl. Llama la atención que el francés conjeture cuestiones propiamente ontológicas teniendo en cuenta que ese periodo es husserliano. Lo relevante de esto es que, a pesar de ser un periodo marcadamente fenomenológico, particularmente en La trascendencia del ego encontramos reflexiones que se corresponden con su segundo periodo, con el llamado periodo heideggeriano. Efectivamente, en el texto de 1934 Sartre se enfrenta a Husserl y no a Heidegger, más aun, siendo más rigurosos, al autor de Ser y tiempo se lo refiere tan solo en una oportunidad, precisamente aludiendo a la idea de "mundo" (Sartre 1968, 51), pero nunca respecto a reflexiones concernientes a su filosofía. Independiente de los periodos

25 En este periodo prolífico, Sartre le solicita a Raymond Aron una sustitución para sumirse en la fenomenología husserliana. Para esto se traslada al Instituto Francés de Berlín por espacio de un año. Ver COHEN-SOLAL, Annie. Sartre 1905-1980. Edhasa, 2005, pp. 133 
del pensamiento de Sartre, de si consideramos, por una parte, la postura de Muñoz que vincula directamente las primeras obras con El ser y la nada, o por otra parte la postura de Cordua que desarticula la obra de Sartre en tres periodos restringidos, no podemos negar que el objetivo fenomenológico que tenía el francés en su primera obra excede su pretensión y se convierte en una obra de existencialismo incipiente. 


\section{Bibliografía}

Cohen-Solal, Annie. 2005. Sartre 1905-1980. España: Edhasa,

González, Encara. 2007. "De la trascendencia del ego al ego trascendental". Revista de filosofía Daimon. N N $^{\circ}$ : 107-118.

Cordua, Carla. 1994. Gerencia del tiempo. Venezuela: Universidad de los Andes.

Deleuze, Gilles. 2009. “La inmanencia, una vida...” en Ensayos sobre Biopolítica, Giorgi y Rodríguez. Argentina: Paidós.

Husserl, Edmund. 1986. Meditaciones Cartesianas. Madrid: Editorial Tecnos.

Husserl, Edmund. 2002. Investigaciones Lógicas, 1. Madrid: Alianza Editorial.

Husserl, Edmund. 2013. Ideas relativas a una fenomenología pura y una filosofía fenomenológica. Libro primero: Introducción general a la fenomenología pura. México: Fondo de Cultura Económica.

Muñoz, José. 1987. Jean Paul Sartre y la dialéctica de la cosificación. Madrid: Editorial Cincel.

Rodríguez, Fernando. 2008. "La trascendencia del ego". Apuntes Filosóficos. Vol. 17. № 32: $39-50$

Sartre, Jean Paul. 1948. Lo imaginario. Buenos Aires: Editorial Iberoamericana.

Sartre, Jean Paul. 1959. Esbozo de una teoría de las emociones. Buenos Aires: Editorial Losada.

Sartre, Jean Paul. 1960. Situaciones I (El hombre y las cosas). Buenos Aires: Editorial Losada.

Sartre, Jean Paul. 1968. La trascendencia del ego. Buenos Aires: Ediciones CALDEN.

Sartre, Jean Paul. 1984. La imaginación. Madrid: Editorial SARPE.

Sartre, Jean Paul. 2008. El ser y la nada. Buenos Aires: Editorial Losada. 